\title{
Exploring multilocus associations of inflammation genes and colorectal cancer risk using hapConstructor
}

\author{
Karen Curtin ${ }^{1 *}$, Roger K Wolff', Jennifer S Herrick', Ryan Abo², Martha L Slattery ${ }^{1}$
}

\begin{abstract}
Background: In candidate-gene association studies of single nucleotide polymorphisms (SNPs), multilocus analyses are frequently of high dimensionality when considering haplotypes or haplotype pairs (diplotypes) and differing modes of expression. Often, while candidate genes are selected based on their biological involvement in a given pathway, little is known about the functionality of SNPs to guide association studies. Investigators face the challenge of exploring multiple SNP models to elucidate which variants, independently or in combination, might be associated with a disease of interest. A data mining module, hapConstructor (freely-available in Genie software) performs systematic construction and association testing of multilocus genotype data in a Monte Carlo framework. Our objective was to assess its utility to guide statistical analyses of haplotypes within a candidate region (or combined genotypes across candidate genes) beyond that offered by a standard logistic regression approach.

Methods: We applied the hapConstructor method to a multilocus investigation of candidate genes involved in pro-inflammatory cytokine IL6 production, IKBKB, IL6, and NFKB1 (16 SNPs total) hypothesized to operate together to alter colorectal cancer risk. Data come from two U.S. multicenter studies, one of colon cancer (1,556 cases and 1,956 matched controls) and one of rectal cancer (754 cases and 959 matched controls).

Results: HapConstrcutor enabled us to identify important associations that were further analyzed in logistic regression models to simultaneously adjust for confounders. The most significant finding (nominal $P=0.0004$; false discovery rate $q=0.037$ ) was a combined genotype association across IKBKB SNP rs5029748 (1 or 2 variant alleles), IL6 rs1800797 (1 or 2 variant alleles), and NFKB1 rs4648110 (2 variant alleles) which conferred an 80\% decreased risk of colon cancer.

Conclusions: Strengths of hapConstructor were: systematic identification of multiple loci within and across genes important in CRC risk; false discovery rate assessment; and efficient guidance of subsequent logistic regression analyses.
\end{abstract}

\section{Background}

In candidate gene association studies, multiple single nucleotide polymorphisms (SNPs) selected to tag variation within a region or chosen based on previous research are usually analyzed independently, in multiSNP haplotypes, and in multiSNP genotype combinations across genes that may be functionally related. Multi-locus analyses are often of high dimensionality, especially when considering whether to analyze

\footnotetext{
* Correspondence: karen.curtin@hsc.utah.edu

'Epidemiology, Department of Internal Medicine, University of Utah Health

Sciences Center, Salt Lake City, Utah, USA

Full list of author information is available at the end of the article
}

haplotype or diplotype data and differing modes of expression, i.e. additive, dominant, or recessive. Studying subsets of SNPs using hapConstructor, freely-available software that performs a systematic and exhaustive construction and testing of multilocus data in a Monte Carlo framework, can guide these analyses to more effectively define the haplotypes or combined genotypes on which susceptibility variants reside [1,2]. As a novel application of the hapConstructor method, we explored multilocus associations in 16 tagging-SNPs across three genes that are functionally related, I-Kappa-B KinaseBeta $(I K B K B)$, Interleukin 6 (IL6), and Nuclear Factor Kappa-B, Subunit 1 (NFKB1) in two multicenter U.S.
() Biomed Central

C 2010 Curtin et al; licensee BioMed Central Ltd. This is an Open Access article distributed under the terms of the Creative Commons Attribution License (http://creativecommons.org/licenses/by/2.0), which permits unrestricted use, distribution, and reproduction in any medium, provided the original work is properly cited. 
case-control studies of colon and rectal cancer. Within these genes, SNPs were genotyped to comprehensively tag genetic variation. Significant single SNP and multiSNP haplotype or combined genotype associations identified in hapConstructor were carried forward to logistic regression models to simultaneously adjust for covariates. The utility of hapConstructor to guide a multilocus investigation is its integrated capability to systematically: infer phase from genotype data, model all possible haplotypes within genes, and model combined genotypes across genes (for differing inheritance modes). Thus, the potentially tedious process of setting up and evaluating all possible multilocus analyses using procedures in statistical software (such as SAS or R) can be avoided.

The inflammatory process is thought to be a key underlying component of colorectal cancer (CRC) that is initiated by pro-inflammatory cytokines such as IL-6 in response to an inflammatory insult. IL-6 stimulates secretion of C-reactive protein, an important biomarker for pro-inflammatory status in several diseases, and polymorphisms in the promoter region have been associated with C-reactive protein circulating levels. We previously reported that variants in IL6, dbSNP ID rs1800795 and rs1800796, were associated with reduced risk of colon cancer among recent aspirin/nonsteroidal anti-inflammatory drug (NSAID) users [3]; the rs1800795 promoter SNP, which is in linkage disequilibrium (LD) with rs1800797 $\left(\mathrm{r}^{2}=0.92\right)$, appeared to be greatest for TP53 mutations while rs1800796 appeared to influence all tumor types among NSAID users. Although $I K B K B$ and NFKB1 polymorphisms have not been studied comprehensively with $C R C$, there is strong evidence of their contribution to the inflammation process. Nuclear transcription factor NF- $\kappa \mathrm{B}$ is involved in regulation of cytokines such as IL-6 [4]. In one study, Landi, et al. evaluated a non-functional polymorphism in NFKB1 and did not observe a significant association with colon cancer [5].

To demonstrate its utility, we applied hapConstructor in a multilocus mining of 4 candidate intronic SNPs in $I K B K B$ : rs2272733, rs3747811, rs5029748, and rs10958713; 5 SNPs in IL6: rs1800796, rs1800797, and rs2069827 (promoter); rs2069840 (intron), and rs2069860 (D162V); and 6 intronic SNPs in NFKB1: rs230510, rs3821958, rs4648090, rs4648110, rs4648127, rs11722146, and rs13117745 to comprehensively identify haplotype and composite genotype subsets of these 16 candidate inflammation-related loci associated with colon or rectal cancer. Using hapConstructor individual and multiSNP results to focus our analyses, we further assessed these associations in multivariate logistic regression models, adjusting for potential confounders, in a large U.S. multicenter study of colon cancer (1,556 cases and
1,956 matched controls) and of rectal cancer (754 cases and 959 matched controls).

\section{Methods \\ Study populations}

Data for the study come from two case-control studies that used identical methods to recruit and interview study participants. Cases of first primary colon cancer (ICD-O 2nd edition codes 18.0, 18.2-18.9) diagnosed between 1 October, 1991 and 30 September, 1994 conducted in the Northern California Kaiser Permanent Medical Care Program (KPMCP), the Wasatch Front area of Utah, and the Twin Cities Metropolitan area of Minnesota were included in the first study. A subsequent study included incident cases diagnosed with a first primary tumor in the rectosigmoid junction or rectum identified between May 1997 and May 2001 in KPMCP and the state of Utah. Case eligibility was determined by the Surveillance Epidemiology and End Results Cancer Registries in Northern California and in Utah and the Minnesota Cancer Surveillance System (colon cancer cases only). Case eligibility included being between 30 and 79 years of age at the time of diagnosis, English speaking, mentally competent to complete the interview, no previous history of CRC, and no known (as indicated on the pathology report or previous medical history) familial adenomatous polyposis, ulcerative colitis, or Crohn's disease. Of cases contacted, 83\% participated at KPMCP, 76\% in Utah, and 67\% in Minnesota. In the rectal cancer study, the participation rates were $75 \%$ of cases from KPCMP and 70\% of cases from Utah. The studies were approved by the University of Utah Institutional Review Board as well as Institutional Review Boards at the Kaiser Permanente Medical Care Program of Northern California (KPMCP) and the University of Minnesota.

Controls, subject to the same eligibility criteria as cases, were frequency matched to cases by sex and by 5 -year age cohort. At KPMCP, controls were randomly selected from membership lists. In Utah, controls 65 years and older were randomly selected from lists provided by the Centers for Medicare and Medicaid Services and controls younger than 65 were randomly selected from driver's license lists. In Minnesota, controls were randomly selected from driver's license lists. Of controls contacted for the colon cancer study, 73\% participated at KPMCP, 53\% participated from Minnesota and 69\% participated from Utah. In the rectal cancer study, participation rates were $70 \%$ for KPMCP and $67 \%$ for Utah. The study populations have been previously described in detail $[6,7]$. Characteristics of colon and rectal cancer study participants are shown in Table 1. 
Table 1 Characteristics of colon cancer and rectal cancer studies

\begin{tabular}{|c|c|c|c|c|c|c|c|c|}
\hline \multirow[b]{3}{*}{ Characteristic } & \multicolumn{4}{|c|}{ Colon study } & \multicolumn{4}{|c|}{ Rectal study } \\
\hline & \multicolumn{2}{|c|}{ Cases } & \multicolumn{2}{|c|}{ Controls } & \multicolumn{2}{|c|}{ Cases } & \multicolumn{2}{|c|}{ Controls } \\
\hline & $\mathbf{n}$ & $\%$ & $\mathrm{n}$ & $\%$ & $\mathbf{n}$ & $\%$ & $\mathbf{n}$ & $\%$ \\
\hline Total & 1556 & & 1956 & & 754 & & 959 & \\
\hline Male & 871 & 56.0 & 1047 & 53.5 & 451 & 59.8 & 541 & 56.4 \\
\hline Female & 685 & 44.0 & 909 & 46.5 & 303 & 40.2 & 418 & 43.6 \\
\hline
\end{tabular}

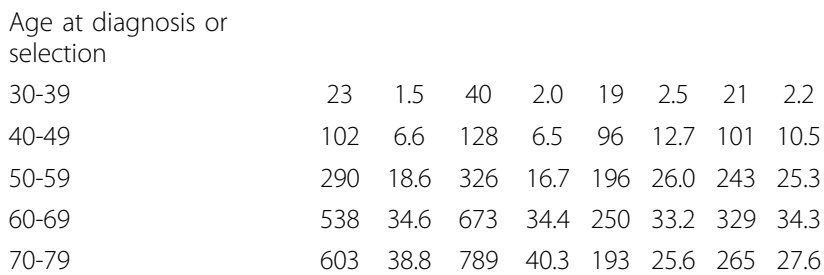

Center

No. California (KPMCP) $\quad 744 \quad 47.8 \quad 787 \quad 40.2 \quad 480 \quad 63.7 \quad 594 \quad 61.9$

$\begin{array}{lllllllll}\text { Utah } & 250 & 16.1 & 378 & 19.3 & 274 & 36.3 & 365 & 38.1\end{array}$

Minnesota

$\begin{array}{lllllllll}562 & 36.1 & 791 & 40.4 & 0 & - & 0 & -\end{array}$

White, non-Hispanic $\quad \begin{array}{lllllllll}1429 & 91.8 & 1828 & 93.5 & 625 & 82.9 & 824 & 85.9\end{array}$

$\begin{array}{llllllllll}\text { White, Hispanic } & 59 & 3.8 & 75 & 3.8 & 61 & 8.1 & 63 & 6.6\end{array}$

$\begin{array}{lllllllll}\text { Black } & 68 & 4.4 & 53 & 2.7 & 29 & 3.8 & 43 & 4.5\end{array}$

$\begin{array}{lllllllll}\text { Asian } & 0 & - & 0 & - & 39 & 5.2 & 29 & 3.0\end{array}$

\section{Data collection}

Trained and certified interviewers collected diet and lifestyle data [8]. The referent year for the study was the calendar year approximately 2 years prior to date of diagnosis (cases) or selection (controls). Information was collected on demographic factors such as age, sex, and study center; diet, physical activity, aspirin and non-steroidal drug use, body size, and other lifestyle factors including medical, family, and reproductive history. Participants were asked to report their race/ethnicity; most reported they were white, non-Hispanic (Table 1). The data collection methods of these studies have been detailed previously $[6,7]$.

\section{Genotyping}

All markers were genotyped using a multiplexed bead array assay format based on GoldenGate chemistry (Illumina, San Diego, California). A genotyping call rate of 99.85\% was attained. Two-hundred-fifty-three internal replicates representing $4.4 \%$ of the sample set were blinded and included. The duplicate sample genotype concordance rate was $100.00 \%$ as determined by 350,070 matching genotypes among sample pairs.

Polymorphisms on the custom Illumina platform were selected to account for genetic variation within each candidate gene with redundant SNPs genotyped in most tagging-SNP linkage disequilibrium bins. Of 7 markers in $I K B K B$, one SNP failed genotyping, one SNP was monomorphic in our study populations, and one SNP failed Hardy-Weinberg equilibrium (HWE) testing resulting in 4 SNPs carried forward to analysis. Of 6 markers in IL6, one SNP (rs1800795) was in high LD with another promoter SNP (rs1800797) and was not carried forward to analysis, resulting in 5 IL6 SNPs. Of 18 NFKB1 markers, one SNP failed genotyping, one very rare SNP had a MAF $<0.01$, and 9 SNPs were redundant and well-represented by another binned taggingSNP with fewer missing values in the data (pairwise $\mathrm{r}^{2}>$ 0.80 ); thus 7 SNPs were carried forward to analysis, for a total of 16 SNPs. A description of genotyped markers (in chromosome order) in IKBKB, IL6, and NFKB1 (including MAF and $P$-HWE) is shown in Additional file 1 . A total of 5,225 samples were successfully genotyped: 1,956 controls and 1,556 cases, colon study; 959 controls and 754 cases, rectal study.

\section{Statistical methods}

The hapConstructor 1.0 module of Genie 2.7.2.1, a freely available software package [2], was used to comprehensively analyze multi-locus SNP haplotypes and combined genotypes to identify associations with affected status and assess their significance in a Monte Carlo framework, as previously detailed [1]. Briefly, all single SNP associations are tested independently. In each subsequent forward step, a SNP is added to twolocus through n-locus SNP sets whose test of association of an empirical $P$-value exceeds a user-defined threshold at the previous step. Rather than specify a significance threshold that remained constant at each step, we decided a priori to use successively more stringent empirical $P$-value significance thresholds (10,000 simulations), as the number of SNPs within each multilocus subset was increased: $0.05,0.01,0.001$ for single, two, and three-locus sets, respectively; and 0.0001 for $\geq 4$ locus sets. First, all single SNPs are tested for association with colon and rectal cancer. For any SNP that reaches a single SNP $P$-value threshold of 0.05 , the second step considers all 2-locus SNP sets containing the significant SNP. Any 2-locus haplotypes or composite genotypes that then surpass a 2-locus threshold of 0.01 are carried forward to a third step which considers all 3-locus SNP sets that include the two markers. Any 3-locus subsets that surpass a threshold of 0.001 are carried forward to an analysis of 4-locus subsets, and so forth until there are no subsets that satisfy a subsequent threshold of 0.0001 . This feature allows the user to alter the significance level at each subsequent step, analogous to data mining procedures used in SNP association studies such as hill climbing that allow the "best" models to move 
forward or a greedy algorithm that performs an optimization $[9,10]$. More stringent $P$ values with each progressive step also assured that multi-locus models were adding statistically beyond significance observed for a single SNP or combinations of SNPs from a previous step, and multilocus models that achieve significance are focused on those more likely to identify a significant haplotype association or interaction across genes after accounting for multiple comparisons. Thus, in our genetic association investigation and in those of others [11-13], we find increasing the stringency of the significance level as the model becomes more complex is appropriate in moving toward a more tractable solution given the large number of comparisons.

For each gene, the full-length haplotype frequencies were estimated from genotype data based on an expectation-maximization algorithm [14], and the maximum likelihood estimate haplotype pairs for each subject were used for analysis. For two-locus haplotype combinations from biallelic data, each set of 4 possible two-locus haplotypes (assuming linkage equilibrium) is modeled as additive, dominant, or recessive (12 haplotype tests). To minimize inferred haplotypes from missing genotypes, samples missing data in $I K B K B, I L 6$, or $N F K B 1$ were excluded from within-gene analyses and samples missing any data for these 3 genes were excluded from analyses across all markers. To avoid sparse cell sizes, hapConstructor examines composite genotypes modeled as dominant and recessive, but not additive. Composite genotype models include all dominant and recessive combinations across loci; assuming linkage equilibrium, 4 composite genotypes can be tested across any two SNPs: dominant/dominant, dominant/recessive, recessive/dominant, or recessive/recessive. If the underlying mode of inheritance is additive, a dominant model performs adequately. Summaries of all tests were stored, and a construction-wide false discovery rate (FDR) that accounts for the multi-SNP process was generated, as described [1]. All $P$-values for individual odds ratio test statistics in hapConstructor were empirically derived based on 10,000 simulations in the Genie null distribution as detailed previously [15]. Although the study population was predominantly white and non-Hispanic, separate genotype files by race/ethnicity to account for allele frequency differences were incorporated using the meta-analysis capabilities of Genie based on CochranMantel-Haenszel techniques as previously detailed [16].

$\mathrm{SAS}^{\circ}$ statistical package, version 9.2 (Cary, N.C.) was used to conduct logistic regression analyses to simultaneously consider covariates. Logistic regression models were used to estimate odds ratios (OR) and 95\% confidence intervals (CI) for associations between independent genotypes and multilocus SNP sets identified as significantly associated in hapConstructor in colon or rectal cancer. Logistic regression models were adjusted for variables used to match controls to cases: age at diagnosis or selection, state of residence (center), sex, and race [17]. Additionally, logistic regression models were adjusted for potential confounders: smoking, use of nonsteriodal anti-inflammatory medications, body mass index, and family history; however, these variables did not substantively impact estimates, and thus were not included. For gene-gene interaction logistic regression models (Additional file 2), $P$ for interaction was determined by comparing a full model including an ordinal multiplicative interaction term to a reduced model without an interaction term, using a likelihood ratio test [18].

\section{Results}

Our findings from hapConstructor and logistic regression analyses are presented as follows: first, associations of single SNPs analyzed independently; second, associations of multilocus haplotypes within each gene; third, associations of multilocus composite genotypes across genes including an illustration of the hapConstructor method and its use in addressing multiple comparisons.

\section{Single SNP results}

In the hapConstructor analysis used to systematically explore associations for haplotypes within IKBKB, IL6, and NFKB1 and composite SNP genotypes across genes, we generally observed similar associations for the same single-locus SNPs significant at the 0.05 level in hapConstructor as for a logistic regression analysis of each SNP considered independently (Table 2). In colon cancer, independent variant alleles in all three genes appear to decrease risk of colon cancer. Conversely, in rectal cancer, only NFKB1 SNPs were independently associated, with 3 of 4 polymorphisms increasing risk. Additional file 1 includes a complete listing of ORs and 95\% CIs from logistic regression models for all independent SNPs in $I K B K B, I L 6$, and NFKB1 and risk of colon and rectal cancer, minimally adjusted for age, sex, race and center.

\section{Haplotype results}

Any SNP identified as significantly associated with colon and rectal cancer in the first step was then analyzed in 2-locus haplotypes with all other markers within $I K B K B$, IL6, and NFKB1. We did not observe any informative haplotypes within $I K B K B$ or IL6 using hapConstructor that were associated with colon cancer beyond the single SNP results, nor in rectal cancer. Using hapConstructor, we were able to identify significant 2-locus NFKB1 haplotypes carried forward to subsequent logistic regression analyses of cancer risk as shown in Table 3 by study, in order of significance. Although hapConstructor is a data 
Table 2 Single SNP results from hapConstructor and from multivariable-adjusted logistic regression

\begin{tabular}{|c|c|c|c|c|c|c|c|}
\hline \multirow[b]{2}{*}{ Gene } & \multirow[b]{2}{*}{ SNP } & \multirow[b]{2}{*}{ Comparison } & \multirow{2}{*}{$\begin{array}{l}\text { Genetic } \\
\text { Model }\end{array}$} & \multicolumn{2}{|c|}{ hapConstructor* } & \multicolumn{2}{|c|}{ Logistic regression ${ }^{* *}$} \\
\hline & & & & OR & $P$ & OR & $95 \% \mathrm{Cl}$ \\
\hline \multicolumn{8}{|c|}{ Colon study } \\
\hline IL6 & rs2069860 & AT/TT vs. AA & Dominant & 0.53 & 0.02 & 0.55 & $0.32,0.95$ \\
\hline$I K B K B$ & rs2272733 & TT vs. CC & Additive & 0.55 & 0.02 & 0.56 & $0.34,0.93$ \\
\hline \multirow[t]{4}{*}{ NFKB1 } & rs4648110 & AA vs. TT & Additive & 0.63 & 0.01 & 0.65 & $0.44,0.94$ \\
\hline & & AA vs. TT/TA & Recessive & 0.64 & 0.01 & 0.66 & $0.45,0.96$ \\
\hline & rs13117745 & TT vs. CC & Additive & 0.61 & 0.03 & 0.61 & $0.37,1.00$ \\
\hline & & TT vs. CC/CT & Recessive & 0.62 & 0.04 & 0.64 & $0.39,1.04$ \\
\hline \multicolumn{8}{|c|}{ Rectal study } \\
\hline \multirow[t]{5}{*}{ NFKB1 } & rs230510 & TT vs. AA & Additive & 0.66 & 0.004 & 0.65 & $0.49,0.87$ \\
\hline & & TT vs. AA/AT & Dominant & 0.77 & 0.009 & 0.79 & $0.51,0.94$ \\
\hline & rs3821958 & GG vs. AA & Additive & 1.34 & 0.04 & 1.32 & $1.00,1.75$ \\
\hline & rs11722146 & GA/AA vs. GG & Dominant & 1.21 & 0.047 & 1.24 & $1.03,1.51$ \\
\hline & rs13117745 & TT vs. CC & Additive & 1.92 & 0.04 & 1.69 & $0.93,3.07$ \\
\hline
\end{tabular}

NOTES: Odds ratios (ORs) compared to reference of major allele homozygotes; $95 \%$ confidence interval $(95 \% \mathrm{Cl})$

*adjusted for race

**adjusted for age, sex, center, and race

mining tool that can systematically assess multilocus associations within candidate genes or regions, a determination by the researcher of the informativeness of any haplotype of interest is nonetheless warranted. In relation to NFKB1 and risk of colon cancer, the most significant single locus identified in hapConstructor was rs4648110 (Table 2). In significant two-locus models, a second SNP, either rs230510 (A) or rs13117745 (T), was carried in a haplotype with the rs4648110 minor allele
(Table 3). However, haplotype ORs did not differ substantively from the effect size of carrying variant rs4648110 or rs13117745 alleles, when the SNP genotypes were considered alone $(P=0.009$; FDR $q=$ $0.187)$. Pairwise correlation between this marker and rs13117745 was $r^{2}=0.71$, and thus may be the same association result with an underlying disease locus. Pairwise correlation between rs4648110 and rs230510 was low $\left(\mathrm{r}^{2}=0.18\right)$, however carriage of the $\mathrm{rs} 230510$ major

Table 3 NFKB1 haplotypes and associations with colon and rectal cancer from hapConstructor and from multivariableadjusted logistic regression

\begin{tabular}{|c|c|c|c|c|c|c|c|}
\hline \multirow[b]{2}{*}{ SNP 1} & \multirow[b]{2}{*}{ SNP 2} & \multirow[b]{2}{*}{ Haplotype } & \multirow{2}{*}{$\begin{array}{l}\text { Genetic } \\
\text { Model }\end{array}$} & \multicolumn{2}{|c|}{ hapConstructor* } & \multicolumn{2}{|c|}{ Logistic regression** } \\
\hline & & & & OR & $P$ & OR & $95 \% \mathrm{Cl}$ \\
\hline \multicolumn{8}{|l|}{ Colon study } \\
\hline rs230510 A > T & rs4648110 T > A & 2 copies A-A vs. 0/1 copy & Rec. & 0.62 & 0.009 & 0.65 & $0.45,0.95$ \\
\hline rs13117745 C > T & $\mathrm{rs} 4648110 \mathrm{~T}>\mathrm{A}$ & 2 copies T-A vs. 0 copies & Additive & 0.62 & 0.009 & 0.65 & $0.44,0.94$ \\
\hline \multicolumn{8}{|l|}{ Rectal study } \\
\hline rs3821958 A > G & rs230510 A > T & 2 copies A-T vs. 0 copies & Additive & 0.66 & 0.006 & 0.68 & $0.50,0.91$ \\
\hline rs4648127 C > T & $\mathrm{rs} 230510 \mathrm{~A}>\mathrm{T}$ & 2 copies C-T vs. 0 copies & Additive & 0.66 & 0.006 & 0.68 & $0.50,0.91$ \\
\hline rs11722146 G > A & $\mathrm{rs} 230510 \mathrm{~A}>\mathrm{T}$ & 2 copies G-T vs. 0 copies & Additive & 0.66 & 0.006 & 0.67 & $0.50,0.90$ \\
\hline rs13117745 C > T & $r s 230510 A>T$ & 2 copies C-T vs. 0 copies & Additive & 0.66 & 0.006 & 0.67 & $0.50,0.90$ \\
\hline$r s 230510 \mathrm{~A}>\mathrm{T}$ & rs4648090 G > A & 2 copies T-G vs. 0 copies & Additive & 0.67 & 0.007 & 0.68 & $0.51,0.90$ \\
\hline rs11722146 G > A & rs4648090 G > A & 2 copies A-A vs. 0 copies & Additive & 1.47 & 0.007 & 1.47 & $1.11,1.93$ \\
\hline$r s 230510 \mathrm{~A}>\mathrm{T}$ & rs4648110 T > A & 2 copies T-T vs. 0 copies & Additive & 0.68 & 0.009 & 0.68 & $0.51,0.91$ \\
\hline rs3821958 A > G & rs4648110 T > A & 2 copies G-A vs. 0 copies & Additive & 1.52 & 0.005 & 1.49 & $1.12,1.99$ \\
\hline rs11722146 G > A & rs13117745 C > T & 2 copies A-T vs. 0 copies & Additive & 1.51 & 0.005 & 1.53 & $1.16,2.01$ \\
\hline rs11722146 G > A & rs4648127 C > T & 2 copies A-T vs. 0 copies & Additive & 1.33 & 0.004 & 1.36 & $1.12,1.65$ \\
\hline rs3821958 A > G & rs13117745 C > T & 2 copies G-T vs. 0 copies & Additive & 1.53 & 0.003 & 1.52 & $1.13,2.02$ \\
\hline rs11722146 G > A & rs4648110 T > A & 2 copies A-A vs. 0 copies & Additive & 1.54 & 0.0028 & 1.55 & $1.17,2.04$ \\
\hline
\end{tabular}

NOTES: Odds ratio (OR); $95 \%$ confidence interval (95\% Cl); Recessive (Rec)

*adjusted for race

**adjusted for age, sex, center, and race; all $P$-trends (additive model) were significant, $<0.01$ 
allele in a haplotype with the rs4648110 variant likewise did not substantively alter risk. Similarly in rectal cancer, the most significantly associated NFKB1 SNP, rs230510, appears to independently contribute to decreased risk with no additional information in terms of either effect size or significance, conferred by a second locus (Tables 2 and 3). The most nominally significant 2-locus haplotype associated with rectal cancer $(P=0.0028$; FDR $q=0.074)$ was across rs11722146 and rs4648110 $\left(\mathrm{r}^{2}=0.10\right)$ in which carrying two copies of the minor alleles A-A confers a $\sim 50 \%$ increased risk, which is somewhat greater in effect than an OR of 1.2 when rs11722146 A allele was considered independently. There were no haplotypes beyond the 2-locus step that were significant at more stringent thresholds.

\section{Composite genotype results}

In complex disease pathways in which it is hypothesized a number of genes of modest effect work together to influence risk, composite genotypes across markers residing on different chromosomes may be important. Similar to hapConstructor steps outlined above for investigating SNP haplotypes within genes, markers across all three genes were analyzed jointly. Any significant 2-SNP composite genotypes in hapConstructor are brought forward into an analysis of 3 SNPs etc., using successively more stringent significance thresholds in each step. We were able to identify genotype combinations that appear to influence risk of colon and rectal cancer using hapConstructor for SNPs across $I K B K B, I L 6$, and NFKB1. In colon cancer, a twoSNP composite genotype comprised of IL6 rs1800797 (dominant model) and NFKB1 rs4648110 (recessive model) was significantly associated with $60 \%$ decreased risk $(P=0.0004$; FDR $q=0.037)$. At the three-locus construction step, a combined genotype also including NFKB1 rs13117745 was of the same magnitude in effect, with a somewhat greater nominal significance $(P=0.0002)$. The presence of LD between the two NFKB1 markers (rs1311745 and rs4648110) in this three-locus set was previously noted. The strongest hapConstructor association (also significant after accounting for multiple tests) was observed across all 3 inflammation-related genes, IKBKB (rs5029748) IL6 (rs1800797), and NFKB1 (rs4648110). Individuals with 1 or 2 minor alleles at rs5029748 and rs1800797, combined with 2 minor alleles at rs4648110, had an almost $80 \%$ decreased colon cancer risk $(P=0.0004$; FDR $q=$ $0.037)$. Although this composite genotype profile was relatively rare in our population ( $1 \%$ of subjects), carriers of major alleles at these common loci appear to be at greater risk of disease. There were no combinations of $\geq 4$ SNPs in colon study participants that met hapConstructor significance thresholds.
In rectal cancer, only two-locus composite genotypes across $I K B K B$ and NFKB1, and IL6 and NFKB1 markers met significance thresholds. The most significant findings were IL6 rs1800797 (dominant model) and either NFKB1 rs11722146 or rs3821958 (also modeled as dominant). However, adjusting for multiple tests the observed significance may represent a chance finding (FDR $q=0.44$ ). In terms of effect size, the odds ratios do not differ substantively from single SNP results although in logistic regression models adjusted for covariates, IKBKB rs3747811 and IL6 rs1800797 may mitigate increased risk of $N F K B 1$. Informative composite genotype models identified in hapConstructor were subsequently analyzed in multivariate-adjusted logistic regression models as highlighted in Table 4. Results of logistic regression models including a multiplicative interaction term for composite genotype associations identified in hapConstructor (Table 4) are shown in Additional file 2 to illustrate the utility of hapConstructor to prescreen significant SNP combinations that inform more complicated analyses.

The hapConstructor method, applied to our analysis of colon risk across genes, is illustrated in Figure 1. In the first step, 4 single SNP associations were identified that met a significance of 0.05 , using a dominant, recessive and additive genotype model for each of 16 SNPs for a total of 47 tests (rs2069860 was not tested using a recessive model because there were no rare-allele homozygotes). These 4 SNPs were carried forward to step 2 and tested in 2-locus composite genotype models with each other (6 comparisons) and all 12 SNPs remaining (48 comparisons). Depending on allele frequencies and the degree of LD between SNPs, not all 4 compositemodel combinations between two loci (dominant/ dominant, dominant/recessive, recessive/dominant, or recessive/recessive) may have data. In our colon cancer analysis, a total of 155 tests were performed at the 2 -locus step out of a possible 216 tests. There were 3 models of 4 SNPs, in 2-locus combinations, significant at the 0.01 level that were carried forward to step 3 . At the 3-locus step, 79 tests were performed out of a possible 312 tests (39 comparisons and a maximum of 8 composite genotype models, various combinations of dominant or recessive at each of 3 SNPs). There were 3 models of 3-locus combinations, involving 5 SNPs, which met a significance threshold of 0.001 and thus were carried forward. At the 4-locus step, 68 tests were performed out of 576 possible tests (36 comparisons and a maximum of 16 composite genotype models). There were no 4-locus results significant below 0.0001, and thus hapConstructor did not continue further. A total of 349 tests were performed in the multilocus analyses of colon cancer across all three genes in hapConstructor as outlined, which resulted in 4 single-SNP 
Table 4 Composite genotype associations with colon and rectal cancer from hapConstructor and from multivariableadjusted logistic regression

\begin{tabular}{|c|c|c|c|c|c|c|c|c|}
\hline \multirow[b]{2}{*}{ SNP 1} & \multirow[b]{2}{*}{ SNP 2} & \multirow[b]{2}{*}{ SNP 3} & \multirow[b]{2}{*}{ SNP1/SNP2/SNP3 genotype } & \multirow{2}{*}{$\begin{array}{l}\text { Genetic } \\
\text { Model }\end{array}$} & \multicolumn{2}{|c|}{ hapConstructor* } & \multicolumn{2}{|c|}{ Logistic regression ${ }^{* *}$} \\
\hline & & & & & OR & $P$ & OR & $95 \% \mathrm{Cl}$ \\
\hline \multicolumn{9}{|l|}{ Colon study } \\
\hline IKBKB rs3747811 & NFKB1 rs4648110 & & $(\mathrm{TA}$ or $\mathrm{AA}) / \mathrm{AA}$ & Dom./Rec. & 0.56 & 0.0094 & 0.54 & $0.34,0.87$ \\
\hline IL6 rs2069827 & NFKB1 rs4648110 & & (GT or TT)/AA & Dom./Rec. & 0.23 & 0.0058 & 0.21 & $0.06,0.74$ \\
\hline IL6 rs1800797 & NFKB1 rs4648110 & & $(\mathrm{GA}$ or $\mathrm{AA}) / \mathrm{AA}$ & Dom./Rec. & 0.41 & 0.0004 & 0.40 & $0.23,0.72$ \\
\hline IKBKB rs5029748 & IL6 rs1800797 & NFKB1 rs4648110 & $(\mathrm{CA}$ or $\mathrm{AA}) /(\mathrm{GA}$ or $\mathrm{AA}) / \mathrm{AA}$ & Dom./Dom./Rec. & 0.21 & 0.0004 & 0.20 & $0.06,0.67$ \\
\hline IL6 rs1800797 & NFKB1 rs4648090 & NFKB1 rs4648110 & $(\mathrm{GA}$ or $\mathrm{AA}) /(\mathrm{GA}$ or $\mathrm{AA}) / \mathrm{AA}$ & Dom./Dom./Rec. & 0.40 & 0.0004 & 0.40 & $0.21,0.73$ \\
\hline IL6 rs1800797 & NFKB1 rs13117745 & NFKB1 rs4648110 & $(\mathrm{GA}$ or $\mathrm{AA}) /(\mathrm{GA}$ or $\mathrm{AA}) / \mathrm{AA}$ & Dom./Dom./Rec. & 0.39 & 0.0002 & 0.39 & $0.21,0.73$ \\
\hline \multicolumn{9}{|l|}{ Rectal study } \\
\hline IKBKB rs3747811 & NFKB1 rs11722146 & & $(\mathrm{TA}$ or $\mathrm{AA}) /(\mathrm{GA}$ or $\mathrm{AA})$ & Dom./Dom. & 1.30 & 0.0096 & 1.11 & $0.83,1.50$ \\
\hline IL6 rs1800797 & NFKB1 rs11722146 & & $(\mathrm{GA}$ or $\mathrm{AA}) /(\mathrm{GA}$ or $\mathrm{AA})$ & Dom./Dom. & 1.35 & 0.0068 & 1.38 & $1.04,1.83$ \\
\hline IL6 rs1800797 & NFKB1 rs3821958 & & $(\mathrm{GA}$ or $\mathrm{AA}) /(\mathrm{AG}$ or $\mathrm{GG})$ & Dom./Dom. & 1.34 & 0.0044 & 1.15 & $0.85,1.56$ \\
\hline
\end{tabular}

NOTES: Odds ratio (OR); $95 \%$ confidence interval (95\%Cl); Dominant (Dom); Recessive (Rec)

*adjusted for race; reference category is all other composite genotypes

**adjusted for age, sex, center, and race; reference category is major allele homozygote (dominant) or heterozygote (recessive) for each SNP

and 6 multiple-SNP genotype models carried forward to a multivariable logistic regression. Absent a freely available and easy to implement systematic tool such as hapConstructor to guide our analysis of this pathway arm, operator effort to similarly step through an exhaustive analysis of possible combinations of loci under different modes of inheritance would be required (Table 5).

Each multistep hapConstructor colon or rectal association haplotype analysis, using marker files stratified by race/ethnicity, required $\sim 30$ minutes overall runtime using Java 1.6 on a Dell Precision T5500 computer with 4 gigabytes of memory, using a Windows Vista Business 64-bit operating system. A composite genotype analysis incorporating all 16 SNPs required $\sim 90$ minutes. Construction-wide $P$-value assessment and determination of an empirical FDR was considerably more time intensive due to generation of 1,000 simulated datasets (each with 10,000 null simulations) which required an additional 15-20 hours to complete for a within-gene haplotype analysis and 3-4 days to complete for the composite genotype analysis across all SNPs.

\section{Discussion}

Proteins encoded by the genes selected for the analysis were hypothesized as working together to influence colorectal cancer risk through an inflammation-related pathway [19]. Binding of pro-inflammatory cytokines, such as IL-6, to their receptors activate the NF- $\kappa$ B complex which in turn activates expression of cytokines and cycloxygenase- 2 . Thus, NF- $\kappa \mathrm{B}$ is an important nuclear transcription factor that regulates a large number of cytokines and is critical for the regulation of inflammation; increased transcription of NF- $\kappa \mathrm{B}$ can increase inflammation and angiogenesis as well as cell survival and growth [20]. I-kappa-B kinase (IKK), which phosphorylates I-kappa-B proteins, is a key regulator of transcriptional activity of the NF- $\kappa \mathrm{B}$ complex [21]; $\mathrm{I} \kappa \mathrm{B}$ proteins are inhibitors of NF- $\kappa \mathrm{B}[20]$. While the importance of the genes in the inflammation pathway has been well documented, our study is the first to comprehensively examine polymorphisms in either $N F K B 1$ or $I K B K B$ to identify functional polymorphisms associated with colorectal cancer.

For independent SNP associations, we observed associations with $I L 6$ and $I K B K B$ in colon cancer but not rectal cancer. NFKB1 SNPs appeared to be important in both cancers, however the direction of risk differed with variant alleles decreasing risk of colon cancer and, other than rs230510, increasing risk of rectal cancer (Table 2). We have previously reported associations in other genetic polymorphisms, potentially modified by lifestyle and environmental factors, that appear to influence colon and rectal cancer differentially [22-24], and thus these results are not surprising. In our hapConstructor analysis, 2-locus haplotypes within the NFKB1 gene were significant beyond single SNP results (Table 3); however, after considering LD between markers and adjusting for multiple comparisons, it is unlikely that a haplotype represents an independent finding beyond associations observed for single NFKB1 SNPs. In regard to composite genotype associations across all 3 hypothesized inflammation-related genes (Table 4), of interest is our observation that promoter variant IL6 rs1800797, while not independently associated, appears to be working in concert with $I K B K B$ and NFKB1 to influence risk of colon and cancer, even after multiple testing is considered. This SNP is in high LD with a SNP in the IL6 regulatory region $(-572 \mathrm{G}>\mathrm{C}$; rs1800795) that was shown to functionally influence IL-6 expression and increase CRP levels [25]; a potential glucocorticoid receptor element exists nearby. As little is known about the 


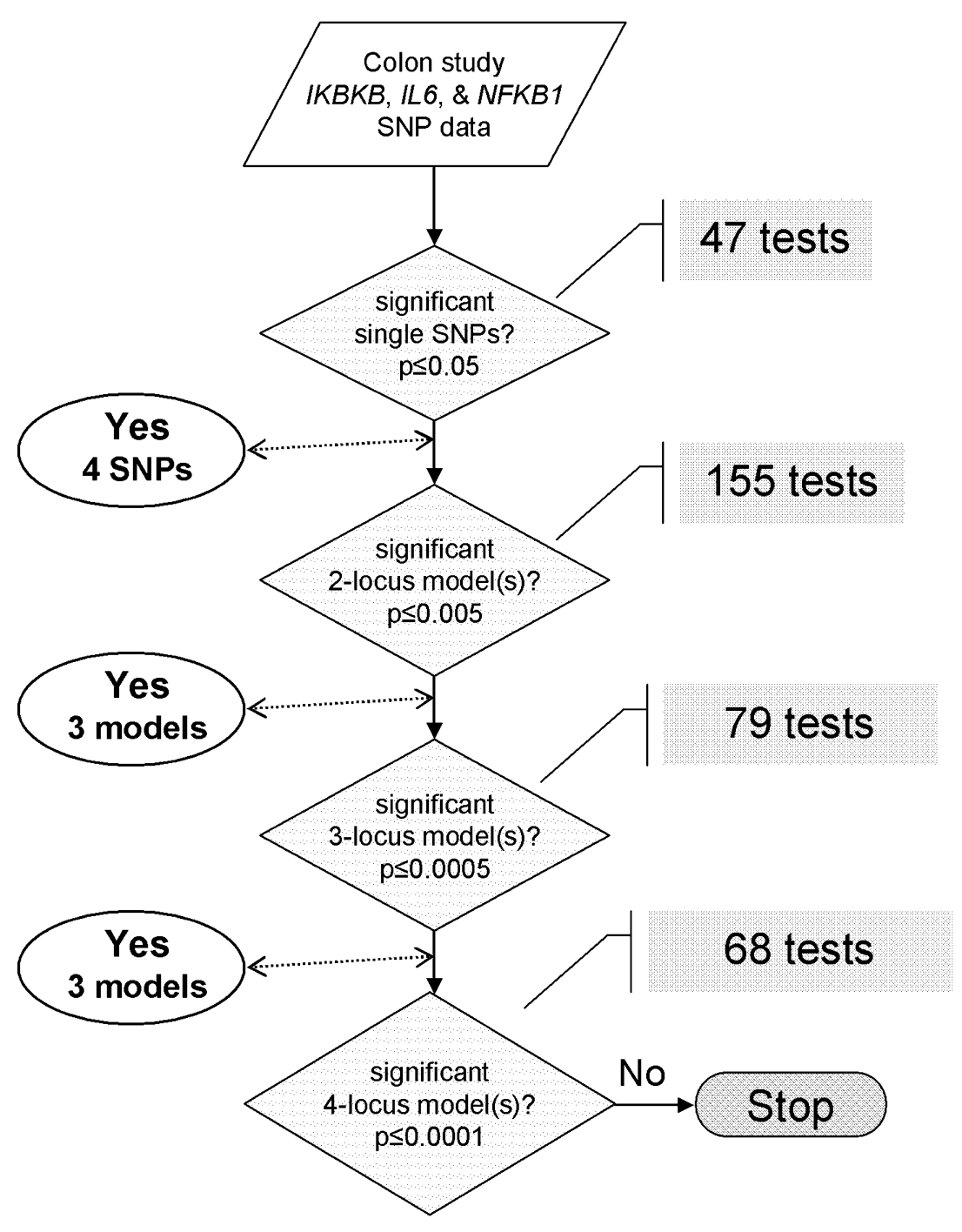

\section{Start} Significant model results

Step 1 1-locus IL6 rs2069860, IKBKB rs2272733, NFKB1 rs4648110 \& rs13117745

Step 2 2-locus IKBKB rs3747811, NFKB1 rs4648110

IL6 rs2069827, NFKB1 rs4648110

IL6 rs1800797, NFKB1 rs4648110

Step 3 3-locus IKBKB rs5029748, IL6 rs1800797, NFKB1 rs4648110

IL6 rs1800797, NFKB1 rs4648090 \& rs4648110

Step 4 4-locus no significant associations

End

Figure 1 Illustration of the hapConstructor method and associations across IKBKB, IL6, and NFKB1 SNPs with risk of colon cancer

biological relevance of SNPs in IKBKB and NFKB1, our findings will be taken forward to functionality testing. In logistic regression analyses of composite genotypes, multiplicative $P$-interaction terms for SNPs across the pathway were significant or borderline significant at the 0.05 level in most models (Additional file 2), which supports the hapConstructor finding of a joint effect.

The hapConstructor tool has been applied to the analysis of haplotypes in recent candidate gene association studies $[12,26,27]$. In addition to haplotypes, an attractive 
Table 5 Comparison of hapConstructor and logistic regression characteristics in multilocus modeling

\begin{tabular}{|c|c|c|c|}
\hline Characteristic & hapConstructor software & $\begin{array}{l}\text { Standard logistic regression } \\
\text { procedure }\end{array}$ & $\begin{array}{l}\text { Stepwise logistic regression } \\
\text { procedure }\end{array}$ \\
\hline $\begin{array}{l}\text { Integrated phase estimation and building of } \\
\text { haplotypes from genotype data }\end{array}$ & Yes & $\begin{array}{l}\text { No; specialized software } \\
\text { required }\end{array}$ & $\begin{array}{l}\text { No; specialized software } \\
\text { required }\end{array}$ \\
\hline $\begin{array}{l}\text { Systematically model all } 2 \text { to n-locus haplotypes within } \\
\text { a gene }\end{array}$ & Yes & $\begin{array}{l}\text { Yes, with additional } \\
\text { programming }\end{array}$ & $\begin{array}{l}\text { Yes, with additional } \\
\text { programming }\end{array}$ \\
\hline $\begin{array}{l}\text { Systematically model mode of inheritance (dominant, } \\
\text { recessive, additive) }\end{array}$ & Yes & $\begin{array}{l}\text { Yes, with additional } \\
\text { programming }\end{array}$ & $\begin{array}{l}\text { Yes, with additional } \\
\text { programming }\end{array}$ \\
\hline $\begin{array}{l}\text { Systematically model all SNP combinations across } \\
\text { genes }\end{array}$ & Yes & No & No \\
\hline User-specified significance levels & $\begin{array}{l}\text { Yes; can vary for single } \\
\text { locus to } \mathrm{n} \text { loci }\end{array}$ & Yes & Yes \\
\hline Model simultaneous covariates & No & Yes & Yes \\
\hline Multiple testing correction & Yes (FDR) & Yes (add-on procedure) & Yes (add-on procedure) \\
\hline $\begin{array}{l}\text { Runtime (excluding additional operator programming } \\
\text { time) }\end{array}$ & Slow to adequate & Fast & Fast \\
\hline
\end{tabular}

feature of hapConstructor is the ability to comprehensively and systematically evaluate composite genotypes of loci across multiple genes (Table 5). This feature is potentially valuable for investigations of multiple genes working together in candidate disease pathways, and our findings represent a novel application of hapConstructor in this regard; furthermore, it is not readily available in standard logistic regression procedures (Table 5). As significant SNP/SNP combinations are carried forward in successive 1 to $n$-locus steps and tested against remaining SNPs, we were able to efficiently capture an important composite gene association including an IL6 promoter SNP (rs1800797) that was not independently associated with colon or rectal cancer and may have otherwise been missed in a traditional logistic regression framework. As participants in our studies differed in race/ethnicity, we were able to utilize the meta-analysis capabilities in Genie software to appropriately assign different allele frequencies to the Monte Carlo simulation of a null distribution [16]. Although our study participants were independent population-based cases and controls, another strength is the ability of hapConstructor in the Genie package to incorporate data of related individuals in families or extended pedigrees instead of, or in addition to, unrelated cases and controls while providing valid significance testing $[15,16]$. Another potential strength is the ability to infer missing genotypes in hapConstructor, as the user can specify the amount of missing data allowed for each sample in haplotype estimation. However, as our analysis also focused on multiSNP associations across genes, inferring missing data from SNPs on other chromosomes was not appropriate and thus samples with missing genotypes were excluded.

A potential limitation of hapConstructor and Monte Carlo testing in general is computational burden which depends on number of SNPs under consideration, number of simulations (particularly in construction-wide assessment of significance and generation of FDR), sample size, and threshold values for significance. In our investigation of 16 loci using 10,000 simulations to provide empirical p-values in 5,225 total cases and controls, hapConstructor runtimes were acceptable, but in other situations may be considerable, depending on the number of genes and SNPs within genes. It is difficult to quantify additional operator time required to set up all possible multilocus haplotype models (within genes) and combined SNP genotype models (across genes) for various modes of inheritance. Although not pre-integrated into standard statistical packages, we acknowledge these capabilities exist but require more programming time for setup and analysis.

A multilocus mining method, hapConstructor is dependent on user-specified significance thresholds in successive 1 to n-locus steps. We chose to specify successively more stringent significance thresholds beginning with a nominal significance of 0.05 for single-locus associations, 0.01 for two-locus associations, 0.001 for three-locus associations, and 0.0001 for 4 or more loci (minimum resolution given 10,000 simulations) to focus on multilocus models that add to statistical significance beyond single SNP associations or SNP combinations identified in a previous step. Using a constant 0.05 threshold, we identified several multilocus associations that met the threshold simply because a single SNP was significant. By using successively more stringent significance levels, we can target models for further investigation that are more likely to identify a true interaction. Although the hapConstructor method can provide an odds ratio statistic and a $P$ for significance, it does not simultaneously control for potential confounding 
variables. We nonetheless found it to be a useful tool to identify multilocus relationships for further analysis in a multivariate logistic regression framework. We believe the integrated capabilities of hapConstructor are most useful when combined with subsequent logistic regression to refine risk estimates and corresponding confidence intervals with adjustment for confounding variables, and to further test for interaction among significant multilocus associations identified in hapConstructor.

The hapConstructor method represents a data mining approach to directing a multiSNP investigation when several genes and SNPs are involved, as in our application. Other methods that reduce the dimensionality of multiSNP analyses have been recently proposed. For example, within a candidate region or gene of interest, Yang, et al. describe a haplotype-based stepwise procedure to eliminate extraneous SNPs in haplotype association analysis [28] and others have proposed principal components regression where multiple SNPs from the candidate region tend to be correlated $[29,30]$. Other methods have been proposed to test for potential genegene interactions to identify specific locus combinations of interest for further investigation, including extended multifactor dimensionality reduction [31] or computationally-efficient algorithms for filtering these interactions [32].

\section{Conclusions}

In conclusion, hapConstructor provided a useful tool to systematically and comprehensively guide our exploration of multilocus SNP combinations in three candidate inflammation-related genes, IKBKB, IL6, and NFKB1 in a U.S. case-control study of colon and rectal cancer. Our findings suggest that polymorphisms in IKBKB, IL6, and NFKB1 may jointly interact to influence colon and rectal cancer risk through an inflammation-related pathway. The utility of hapConstructor was further enhanced by the capability of false discovery rate assessment to account for multiple comparisons.

\section{Additional material}

Additional file 1: Description of candidate SNPs and association with colon or rectal cancer. All genotyped SNPs (in chromosome order) in $\mathbb{K} B K B, \| L 6$, and $N F K B 1$ including their minor allele frequencies and individual odds ratios $(95 \% \mathrm{Cls})$ from logistic regression models for risk of colon and rectal cancer.

Additional file 2: Composite genotype logistic regression model associations with colon and rectal cancer identified in

hapConstructor. Results of logistic regression models including a

multiplicative interaction term for composite genotype associations identified in hapConstructor.

\section{Acknowledgements}

We would like to acknowledge the contributions to this study of John D. Potter, Bette J. Caan, Sandra Edwards, Roger Edwards, and Leslie Palmer for data collection efforts; Michael D. Hoffman and Erica C. Wolff for their contribution in the genetic laboratory; Kenneth Boucher for biostatistical consulting; and Nicola J. Camp for her suggestions and advice in regard to the Genie package.

This study was funded by National Institutes of Health grant R01-CA48998 (to MLS). This research was supported by the Utah Cancer Registry, which is funded by Contract N01-PC-35141 from the National Cancer Institute's Surveillance, Epidemiology and End Results program with additional support from the Utah State Department of Health and the Northern California Tumor Registry. The contents of this manuscript are solely the responsibility of the authors, and do not necessarily represent the official view of the National Cancer Institute.

\section{Author details}

${ }^{1}$ Epidemiology, Department of Internal Medicine, University of Utah Health Sciences Center, Salt Lake City, Utah, USA. ${ }^{2}$ Genetic Epidemiology, Department of Internal Medicine, University of Utah Health Sciences Center Salt Lake City, Utah, USA

\section{Authors' contributions}

KC carried out the statistical analyses and drafted the manuscript. RKW directed the genotyping, and helped to draft the manuscript. JSH provided data management and statistical programming efforts, and helped to draft the manuscript. RA provided technical assistance with software and helped to draft the manuscript. MLS conceived the study, directed its design, coordination, and data collection efforts, and helped to draft the manuscript. All authors read and approved the final manuscript.

\section{Competing interests}

The authors declare that they have no competing interests.

Received: 13 November 2009 Accepted: 3 December 2010

Published: 3 December 2010

References

1. Abo R, Knight S, Wong J, Cox A, Camp NJ: hapConstructor: automatic construction and testing of haplotypes in a Monte Carlo framework. Bioinformatics (Oxford, England) 2008, 24(18):2105-2107.

2. hapConstructor Home Page. [http://www-genepi.med.utah.edu/Genie/ hapConstructor.html].

3. Slattery ML, Wolff RK, Herrick JS, Caan BJ, Potter JD: IL6 genotypes and colon and rectal cancer. Cancer Causes Control 2007, 18(10):1095-1105.

4. Hussain SP, Harris CC: Inflammation and cancer: an ancient link with novel potentials. International journal of cancer 2007, 121(11):2373-2380

5. Landi S, Moreno V, Gioia-Patricola L, Guino E, Navarro M, de Oca J, Capella G, Canzian F: Association of common polymorphisms in inflammatory genes interleukin (IL)6, IL8, tumor necrosis factor alpha, NFKB1, and peroxisome proliferator-activated receptor gamma with colorectal cancer. Cancer Res 2003, 63(13):3560-3566.

6. Slattery ML, Edwards S, Curtin K, Schaffer D, Neuhausen S: Associations between smoking, passive smoking, GSTM-1, NAT2, and rectal cancer. Cancer Epidemiol Biomarkers Prev 2003, 12(9):882-889.

7. Slattery ML, Potter J, Caan B, Edwards S, Coates A, Ma KN, Berry TD: Energy balance and colon cancer-beyond physical activity. Cancer Res 1997, 57(1):75-80.

8. Edwards S, Slattery ML, Mori M, Berry TD, Caan BJ, Palmer P, Potter JD: Objective system for interviewer performance evaluation for use in epidemiologic studies. American journal of epidemiology 1994, 140(11):1020-1028.

9. Zhang $P$, Sheng $H$, Uehara $R$ : A double classification tree search algorithm for index SNP selection. BMC Bioinformatics 2004, 5:89.

10. Laramie JM, Wilk JB, DeStefano AL, Myers RH: HaploBuild: an algorithm to construct non-contiguous associated haplotypes in family based genetic studies. Bioinformatics 2007, 23(16):2190-2192. 
11. Piccolo SR, Abo RP, Allen-Brady K, Camp NJ, Knight S, Anderson JL, Horne BD: Evaluation of genetic risk scores for lipid levels using genome-wide markers in the Framingham Heart Study. BMC proceedings 2009, 3(Suppl 7):S46

12. Shephard ND, Abo R, Rigas SH, Frank B, Lin WY, Brock IW, Shippen A, Balasubramanian SP, Reed MW, Bartram CR, et al: A breast cancer risk haplotype in the caspase-8 gene. Cancer Res 2009, 69(7):2724-2728.

13. Slattery ML, Herrick JS, Lundgreen A, Fitzpatrick FA, Curtin K, Wolff RK: Genetic variation in a metabolic signaling pathway and colon and rectal cancer risk: mTOR, PTEN, STK11, RPKAA1, PRKAG2, TSC1, TSC2, PI3K and Akt1. Carcinogenesis 31(9):1604-1611.

14. Thomas A: GCHap: fast MLEs for haplotype frequencies by gene counting. Bioinformatics 2003, 19(15):2002-2003.

15. Allen-Brady K, Wong J, Camp NJ: PedGenie: an analysis approach for genetic association testing in extended pedigrees and genealogies of arbitrary size. BMC Bioinformatics 2006, 7:209.

16. Curtin K, Wong J, Allen-Brady K, Camp NJ: PedGenie: meta genetic association testing in mixed family and case-control designs. BMC Bioinformatics 2007, 8:448.

17. Bland JM, Altman DG: Matching. Bmj 1994, 309(6962):1128.

18. Agresti A: An introduction to categorical data analysis New York: John Wiley \& Sons, Inc; 1996.

19. Lawrence T, Willoughby DA, Gilroy DW: Anti-inflammatory lipid mediators and insights into the resolution of inflammation. Nat Rev Immunol 2002, 2(10):787-795.

20. Kandel ES: NFkappaB inhibition and more: a side-by-side comparison of the inhibitors of IKK and proteasome. Cell cycle (Georgetown, Tex 2009, 8(12):1819-1820

21. Parker KM, Ma MH, Manyak S, Altamirano CV, Tang YM, Frantzen $M$ Mikail A, Roussos E, Sjak-Shie N, Vescio RA, et al: Identification of polymorphisms of the IkappaBalpha gene associated with an increased risk of multiple myeloma. Cancer genetics and cytogenetics 2002, 137(1):43-48.

22. Slattery ML, Herrick J, Wolff RK, Caan BJ, Potter JD, Sweeney C: CDX2 VDR polymorphism and colorectal cancer. Cancer Epidemiol Biomarkers Prev 2007, 16(12):2752-2755

23. Slattery ML, Murtaugh M, Caan B, Ma KN, Neuhausen S, Samowitz W: Energy balance, insulin-related genes and risk of colon and rectal cancer. International journal of cancer 2005, 115(1):148-154.

24. Slattery ML, Neuhausen SL, Hoffman M, Caan B, Curtin K, Ma KN, Samowitz W: Dietary calcium, vitamin D, VDR genotypes and colorectal cancer. International journal of cancer 2004, 111(5):750-756.

25. Ferrari SL, Ahn-Luong L, Garnero P, Humphries SE, Greenspan SL: Two promoter polymorphisms regulating interleukin- 6 gene expression are associated with circulating levels of C-reactive protein and markers of bone resorption in postmenopausal women. The Journal of clinical endocrinology and metabolism 2003, 88(1):255-259.

26. Curtin K, Lin WY, George R, Katory M, Shorto J, Cannon-Albright LA, Bishop DT, Cox A, Camp NJ: Meta association of colorectal cancer confirms risk alleles at 8q24 and 18q21. Cancer Epidemiol Biomarkers Prev 2009, 18(2):616-621.

27. Curtin K, Lin WY, George R, Katory M, Shorto J, Cannon-Albright LA, Smith G, Bishop DT, Cox A, Camp NJ: Genetic variants in XRCC2: new insights into colorectal cancer tumorigenesis. Cancer Epidemiol Biomarkers Prev 2009, 18(9):2476-2484.

28. Yang Y, Li SS, Chien JW, Andriesen J, Zhao LP: A systematic search for SNPs/haplotypes associated with disease phenotypes using a haplotypebased stepwise procedure. BMC Genet 2008, 9:90.

29. Wang K, Abbott D: A principal components regression approach to multilocus genetic association studies. Genet Epidemiol 2008, 32(2):108-118

30. Gauderman WJ, Murcray C, Gilliland F, Conti DV: Testing association between disease and multiple SNPs in a candidate gene. Genet Epidemiol 2007, 31(5):383-395.

31. Mei H, Ma D, Ashley-Koch A, Martin ER: Extension of multifactor dimensionality reduction for identifying multilocus effects in the GAW14 simulated data. BMC Genet 2005, 6(Suppl 1):S145.

32. Greene CS, Penrod NM, Kiralis J, Moore JH: Spatially Uniform ReliefF (SURF) for Computationally-Efficient Filtering of Gene-Gene Interactions. BioData mining 2009, 2(1):5.

\section{Pre-publication history}

The pre-publication history for this paper can be accessed here: http://www.biomedcentral.com/1471-2350/11/170/prepub

doi:10.1186/1471-2350-11-170

Cite this article as: Curtin et al:: Exploring multilocus associations of inflammation genes and colorectal cancer risk using hapConstructor. BMC Medical Genetics 2010 11:170.

\section{Submit your next manuscript to BioMed Central and take full advantage of:}

- Convenient online submission

- Thorough peer review

- No space constraints or color figure charges

- Immediate publication on acceptance

- Inclusion in PubMed, CAS, Scopus and Google Scholar

- Research which is freely available for redistribution

Submit your manuscript at www.biomedcentral.com/submit
Biomed Central 